\title{
Integrity and quality assessment applied on laser welded titanium components
}

\author{
Håkan Wirdelius ${ }^{\mathrm{a}}$, Kenneth Hamberg and Erik Lindgren \\ SCeNDT/MMT, Chalmers University of Technology, SE-412 96 Göteborg, Sweden
}

\begin{abstract}
Laser welding of thin titanium components, a critical component of many gas turbine engines, has demonstrated a tendency of generating pores in clusters with a prescribed orientation. These pores, also known as chain porosities, are often of harmless sizes (of 50-100 micrometer) as individuals. Though the cluster as such, depending on the distances and orientations in between the pores, may have an impact on the structural integrity. A recently developed algorithm for 3-D positioning of small pore defects in planar geometries using digital X-ray inspection aims at providing 3-D positions of the defects. This could then be used in-line to assess the welding quality in the manufacturing process. This presentation describes the development of a methodology that aims to incorporate nondestructive evaluation with, in this case, structural integrity.
\end{abstract}

\section{Introduction}

Within the nuclear and aeronautic industry advanced forms of non-destructive testing (NDT) methods have been applied both for manufacturing control (e.g. welding) and in-service inspections since more than three decades. This development has been driven by the introduction of structural design and risk based inspection programs based on the damage tolerance concept [1]. Increasing fuel costs and environmental requirements in the air transport industry drives the development of lighter constructions. These aspects have focused the development of non-destructive testing (NDT) technologies to detect possible initialization of in-service defects at an early stage already in the manufacturing process [2].

Laser welding of thin titanium components, a critical component of many jet-engines, has demonstrated a tendency of generating pores in clusters with a prescribed orientation [3]. These pores, sometimes referred to as chain porosities, are often of harmless sizes $(\sim 100$ micrometer $)$ as individuals. However, a cluster as such, depending on the distances and orientations in between the pores, may have an impact on the structural integrity [4]. Usually conventional radiography is applied which provides a two dimensional projection of the three dimensional information and the estimated distance between the pores, if even detected, becomes very conservative by nature [5,6]. A recently developed algorithm for three dimensional positioning of small pore defects in planar geometries using digital X-ray inspection has indicated that it is possible to retrieve the three dimensional position

\footnotetext{
${ }^{a}$ Corresponding author: hakan.wirdelius@chalmers.se
}

This is an Open Access article distributed under the terms of the Creative Commons Attribution License 4.0, which permits unrestricted use, distribution, and reproduction in any medium, provided the original work is properly cited. 

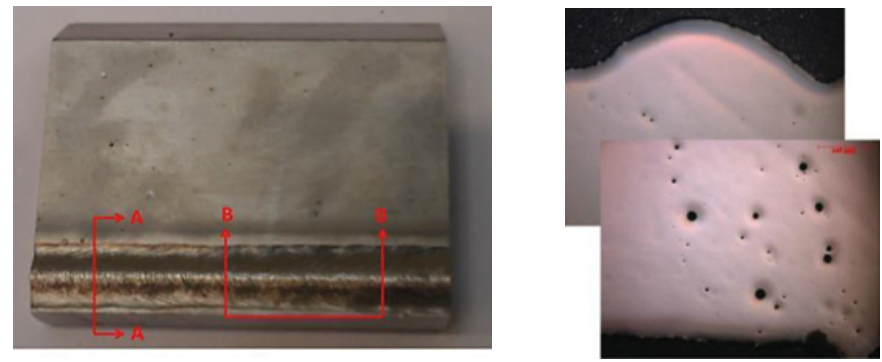

Figure 1. Weld on a titanium plate. The picture to the right shows an example of a cut A-A, the sample is not etched. The picture to the left shows how the various cuts length wise the weld is laid.

of the defects at the manufacturing quality control step [7]. The algorithm and the accompanying inspection procedure is based on taking X-ray projections of the object at a small set of rotation angles. Deterministic simulations based on statistical distributions of defects either assumed $[8,9]$ or based on 3D reconstructions have been described in literature [10].

This contribution intends to describe the development of a methodology that incorporates nondestructive evaluation with structural integrity and thereby providing lifetime assessment. This new area of research, Integrity and quality assessment by NDE (IqNDE), intends to increase the amount of information from the output of non-destructive techniques in order to increase the economic value of the inspections.

A number of thin laser welded titanium components will be produced and investigated with a high resolution X-ray technique. Fatigue tests of components with identified chain porosities will be conducted. Based on the result an investigation of whether the defect information should be transferred into a statistical description and be used as input into statistical fracture mechanics or if instead 3D LEFM should preferably be used to predict the lifetime based on data with well specified uncertainties (Probability of detection, POD) of the used NDT technique [1].

\section{Small scale pores}

Preliminary destructive examinations based on information using high resolution X-ray technique have been conducted. The A-A and the B-B cuts in Fig. 1 are selected in order to identify whether the porosities have any preferred orientation which could be transformed into some statistical description.

A first preliminary evaluation gives that the coarse porosities are in the middle of the weld and the small inclusions are preferably close to the heat affected zone but in the fusion zone. Evaluation of the etched samples show that small gas porosities seems to be guided by the growth of the primary crystals close to the fusion zone and the coarser porosities follow the thermal gradient that force them to the centre of the weld.

\section{References}

[1] J.T.P. Yao, F. Kozin, Y.K. Wen, J.N. Yang, G.I. Schueller, O. Ditlevsen, Structural Safety 3, 231267 (1986)

[2] E. Lindgren, H. Wirdelius, ISABE (2011)

[3] Y. Chen, S. Chen, L. Li, Int. J. Adv. Manuf. Technol. 44, 265-272 (2009)

[4] Y. Murakami, T. Endo, Int. J. of Fatigue 2(1), 23-30, (1980)

[5] Y. Murakami, T. Endo, Int. J. of Fatigue 16(3), 163-182, (1994) 
[6] Y. Murakami, Int. J. of Fatigue 41, 2-10, (2012)

[7] E. Lindgren, EURASIP Journal on Advances in Signal Processing, pp 9 (2014)

[8] D.L. McDowell, Mat. Sc. And Eng. A 468-470, 4-14, (2007)

[9] D.L. McDowell, F.P.E. Dunne, Int. J. of Fatigue 32, 1521-1542, (2010)

[10] M. Shirani, G. Härkegård, Int. J. of Fatigue 41, 188-198 (2012) 